\title{
Soft Bigram distance for names matching
}

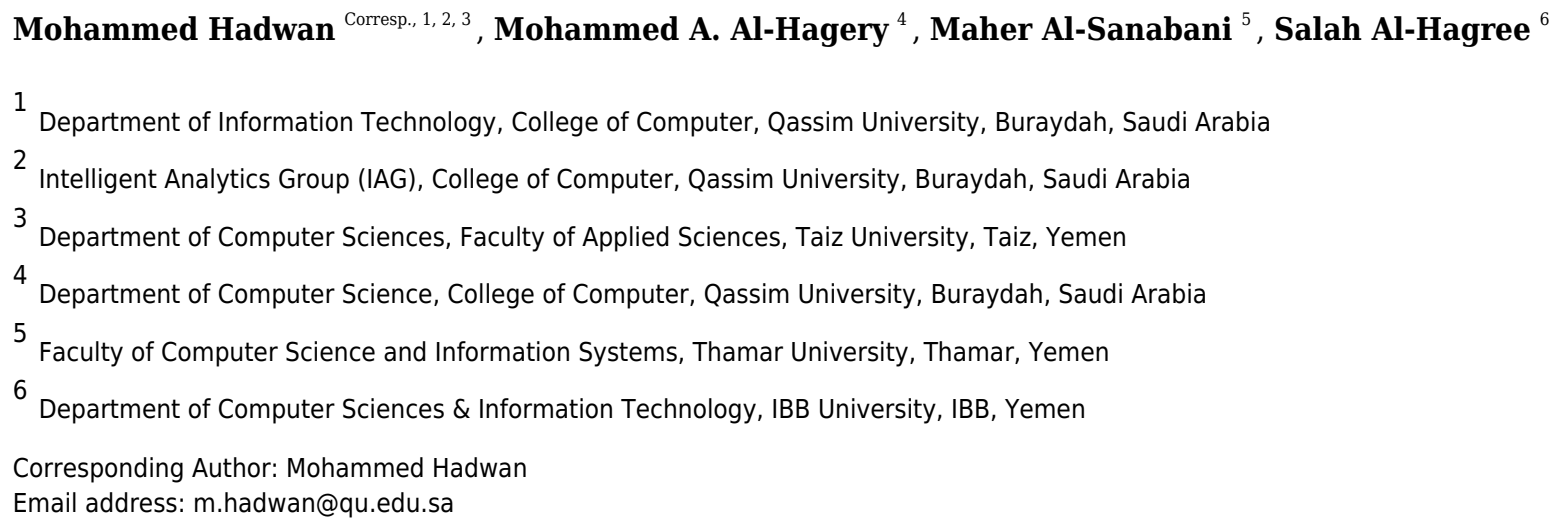

Background: Bi-gram distance (BI-DIST) is a recent approach to measure the distance between two strings that have an important role in a wide range of applications in various areas. The importance of BI-DIST is due to its representational and computational efficiency, which has led to extensive research to further enhance its efficiency. However, developing an algorithm that can measure the distance of strings accurately and efficiently has posed a major challenge to many developers. Consequently, this research aims to design an algorithm that can match the names accurately.BI-DIST distance is considered the best orthographic measure for names identification; nevertheless, it lacks a distance scale between the name bigrams. Methods: In this research, the Soft Bigram Distance (Soft-Bidist) measure is proposed. It is an extension of BI-DIST by softening the scale of comparison among the name Bigrams for improving the name matching. Different datasets are used to demonstrate the efficiency of the proposed method. Results: The results show that Soft-Bidist outperforms the compared algorithms using different name matching datasets. 


\section{Soft Bigram Distance for Names Matching}

3

4

5

6

7 Hagree $^{6}$

$8{ }^{1}$ Department of Information Technology, College of Computer, Qassim University, Buraydah, 9 Saudi Arabia

10 2Department of Computer Science, Faculty of Applied Sciences, Taiz University, Taiz, Yemen

$11{ }^{3}$ Intelligent Analytics Group (IAG), College of Computer, Qassim University, Buraydah, Saudi

12 Arabia

$13{ }^{4}$ Department of Computer Science, College of Computer, Qassim University, Buraydah, Saudi

14 Arabia

15

${ }^{5}$ Faculty of Computer Science and Information Systems, Thamar University, Thamar, Yemen

${ }^{6}$ Department of Computer Sciences \& Information Technology, IBB University, IBB, Yemen

Corresponding Author: Mohammed Hadwan

Email address: M.hadwan@qu.edu.sa

\section{Abstract}

Background: Bi-gram distance (BI-DIST) is a recent approach to measure the distance between two strings that have an important role in a wide range of applications in various areas. The importance of BI-DIST is due to its representational and computational efficiency, which has led to extensive research to further enhance its efficiency. However, developing an algorithm that can measure the distance of strings accurately and efficiently has posed a major challenge to many developers. Consequently, this research aims to design an algorithm that can match the names accurately.BI-DIST distance is considered the best orthographic measure for names identification; nevertheless, it lacks a distance scale between the name bigrams.

Methods: In this research, the Soft Bigram Distance (Soft-Bidist) measure is proposed. It is an extension of BI-DIST by softening the scale of comparison among the name Bigrams for improving the name matching. Different datasets are used to demonstrate the efficiency of the proposed method.

Results: The results show that Soft-Bidist outperforms the compared algorithms using different name matching datasets. 


\section{Introduction}

38 Currently, Name Matching is one of the hottest topics in the emerging data science area, where,

39

40

41

42

43

44

45

46

47

48

49

50

51

52

53

54

55

56

57

58

59

60

61

62

63

64

65

66

67

68

69

70

71

72

73

74

75 the BI-DIST is a recent and significant approach for name matching by measuring the distance between two strings, which play an important role in a wide range of applications in different fields.

Consequently, this led us to develop a strong and effective method for this purpose. Although,., developing highly accurate name matching algorithms is still a challenging issue in the research community (Navarro 2001)(Hall and Dowling 1980). By deeply reviewing the previous studies, it found that several studies have been conducted to develop name-matching algorithms, which are used to cope with many important topics. The classification of these algorithms is implemented into two categories: approximate string matching (inexact) algorithms (Al-Ssulami 2015; Hall and Dowling 1980; Navarro 2001) and exact string-matching algorithms (Al-Ssulami 2015; Charras and Lecroq 2004; Peter Christen 2006).

Name identification and matching are increasingly used in several applications such as Customer Relation Management (CRM), Health Care (HC), Customer Data Integration (CDI), Anti-Money Laundering (AML), Criminal Investigation (CI) and Genealogy Services (GS) (Lisbach et al. 2013). Besides, it is used also in other applications in the airports, Plagiarism Checking software, etc. If the matching is carried out considering only the exact similarity in such applications, it would be difficult and might be impossible to deal with the case of name variations, which is an unavoidable situation when dealing with real-world data sets (Delgado et al. 2016). That is, the exact matching approach is not suitable for large-scale applications and complex information systems, since it cannot retrieve names that have more than one acceptable spelling (Peter Christen 2006).

To have a highly effective name matching methods, the approximate string-matching approach should be adopted rather than exact matching. Therefore, this paper aims to develop an algorithm for name matching, that consider an approximate string-matching algorithm to allow dealing with possible technical or computational errors. Such matching algorithms have been used in several applications such as Spelling correction (Park et al. 2020), Linking database (Hand and Christen 2018), Text retrieval (Abdulhayoglu, Thijs, and Jeuris 2016), Handwriting recognition (Chowdhury, Bhattacharya, and Parui 2013), Computational biology "DNA"(Berger, Waterman, and Yu 2020), and Name recognition (Delgado et al. 2016)... etc. Consequently, in this work, a new softened distance measure is proposed, based on the BI-DIST distance to increase the efficiency and accuracy of the name-matching method. This is achieved by identifying different cases that form bigram scales, grounded on statistical analysis to soften the distance scale. Accordingly, it is hypothesized that an evolutionary method can be adapted to adjust the weights of the distance scale between n-grams. 


\section{Background and related work}

77

78

79

80

81

82

83

84

85

86

87

88

89

90

91

92

93

94

95

96

97

98

99

100

101

102

103

104

105

106

Many research works mainly concentrate on name matching methods improvement and algorithm complexity. In addition to the complex process of matching names as aforementioned, misspelling and different spelling of words are detected. The effective way is to apply an approximate stringmatching technique to prevent the recurring of different spelling inputs and misspelling (Lertnattee and Paluekpet 2019). Given two names $\mathrm{X}$ and $\mathrm{Y}$ represented as strings of $\mathrm{n}$ and $\mathrm{m}$ characters, respectively, the Edit Distance, aka Levenshtein Distance (LD), indicates the least possible cost of editing processes (insertion, deletion, and substitution) to convert X to Y (Levenshtein 1966). For example, if $\mathrm{X}=$ "Zantac\|" and $\mathrm{Y}=$ "Xanax\|", the edit distance is 3 as the minimum transformation implies two substitution operations ("Z" $\rightarrow$ " $X$ " and "c" $\rightarrow$ " $\mathrm{X}$ ") and one deletion operation (letter "t"). Which is calculated using the recurrence formula in Eq. (1), The Levenshtein distance between two strings $s, t$ is given mathematically by $\operatorname{Lev}_{s, t}(|s|,|t|)$ where.

89

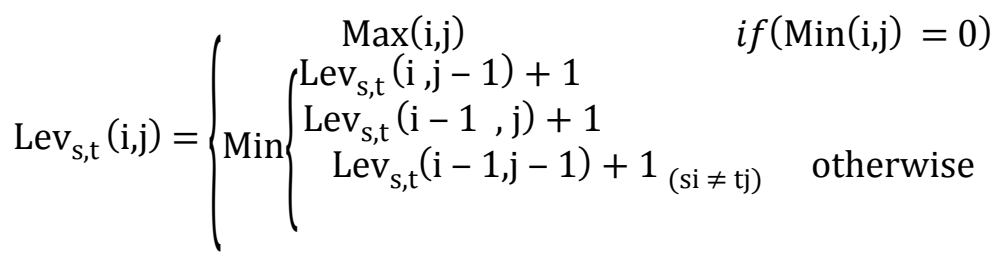

In equation (1), 1 is the indicator function equal to 0 if $\mathrm{s}_{\mathrm{i}==} \mathrm{t}_{\mathrm{j}}$ and 1 otherwise. By $|s|$ we denote the length of the string $\mathrm{s} . \operatorname{Lev}_{\mathrm{s}, \mathrm{t}}(i, j)$ is the distance between string prefixes - the first $\mathrm{i}$ characters of $s$ and the first $\mathrm{j}$ characters of $\mathrm{t}$. The first part of this formula denotes the number of insertion or deletion steps to transform prefix into an empty string or vice versa. The second block is a recursive expression with the first line represents deletion and the second one represents insertion. The last line is responsible for substitutions. More details are available at ${ }^{1}$.

In (Damerau 1964), Damerau-Levenshtein Distance (DLD) is presented which is akin to the LD algorithm. The chief modification is that DLD lets one more edit, particularly where the two adjacent characters can be transposed. The DLD algorithm describes the distance between two strings $\mathrm{s}$ and $\mathrm{t}$ by the following recursive relation as shown in Eq. (2):

$$
\operatorname{DLev}_{\mathrm{s}, \mathrm{t}}(\mathrm{i}, \mathrm{j})=\operatorname{Min} \begin{cases}0 & \text { if } i=j=0 \\ \operatorname{DLev}_{\mathrm{s}, \mathrm{t}}(\mathrm{i}-1, \mathrm{j})+1 & \text { if } i>0 \\ \operatorname{DLev}_{\mathrm{s}, \mathrm{t}}(\mathrm{i}, \mathrm{j}-1)+1 & \text { if } j>0 \\ \operatorname{DLev}_{\mathrm{s}, \mathrm{t}}(\mathrm{i}-1, \mathrm{j}-1)+1(\mathrm{si} \neq \mathrm{t}) & \text { if } i, j>0 \\ \operatorname{DLev}_{\mathrm{s}, \mathrm{t}}(\mathrm{i}-2, \mathrm{j}-2)+1 \text { if } i, j>1 \text { and } s[i]=t[j-1] \text { and } s[i-1]=t[j]\end{cases}
$$

Where $1_{(s i \neq t j)}$ is the indicator function equal to 0 when $s i=t j$ and equal to 1 otherwise.

${ }^{1}$ https://www.baeldung.com/cs/levenshtein-distance-computation 
107

108

109

110

111

112

113

114

115

116

117

118

119

120

121

122

123

124

125

126

127

128

129

130

131

132

133

134

135

136

\section{7}

138

139

140

In (Rees 2014), a customized approach called a Modified Damerau-Levenshtein Distance algorithm (MDLD) was proposed. MDLD was adjusted and tested against two input strings that support block transpositions of numerous characters. The MDLD algorithm's time complex $\mathrm{O}\left(\mathrm{n}^{3}\right)$, is presented algorithm (MDLD) in its Oracle PL/SQL form. More details are available at ${ }^{2}$

The N-gram Distance (N-DIST) that was proposed by Kondrak (Kondrak 2005) in his research works by the fusion of features carried out by grams of size and non-crossing-links constraints, and the first letter is repeated initially. On the other hand, it is found that BI-DIST is a case of NDIST(Kondrak 2005). In (Abdulhayoglu et al. 2016) each matrix element $N D I S T_{\mathrm{s}, \mathrm{t}}(\mathrm{i}, \mathrm{j})$ is calculated according to Eq. (3), where the cost in Eq. (4) is the total number of distinct letters in the same positions in the character $n$-grams $\mathrm{s}_{\mathrm{i}} ; \mathrm{t}_{\mathrm{j}}$, and $\mathrm{n}$ is the size of the character $\mathrm{n}$-gram, as shown in Eqs. (3)-(4):

(1)

$$
\begin{aligned}
& N D I S T_{\mathrm{s}, \mathrm{t}}(\mathrm{i}, \mathrm{j})=\left\{\begin{array}{l}
\operatorname{Max}(\mathrm{i}, \mathrm{j}) \\
\operatorname{Min}\left\{\begin{array}{l}
N D I S T_{\mathrm{s}, \mathrm{t}}(\mathrm{i}-1, \mathrm{j})+1 \\
N D I S T_{\mathrm{s}, \mathrm{t}}(\mathrm{i}, \mathrm{j}-1)+1 \\
N D I S T_{\mathrm{s}, \mathrm{t}}(\mathrm{i}-1, \mathrm{j}-1)+d_{n}\left(T_{i, j}^{n}\right) .
\end{array} \quad(\mathrm{i}=0 \text { or } \mathrm{j}=0)\right.
\end{array}\right. \\
& d_{n}\left(T_{i, j}^{n}\right)=\frac{1}{n} \sum_{u=1}^{n} d_{1}\left(x_{i+u}, y_{j+u}\right),
\end{aligned}
$$

Kondrak (Kondrak 2005) proposed the measures N-gram Distance and Similarity (N-DIST and N-SIM) respectively, where the recall metric is used to assess the results of twelve measures with the U.S. Pharmacopeia (USP) look-alike/sound-alike (LASA)list of 360 unique drug names. In this study, Kondrak concluded that combining BI-DIST and BI-SIM achieves the best results. The Food and Drug Administration (FDA) uses it to create automated warning systems to identify potential LASA errors in prescription electronic systems and phonetic orthographic computer analysis (POCA) software. Moreover, (Millán-Hernández et al. 2019) proposed a Soften Bigram Similarity measure (Soft-Bisim). This work concentrated on improving an algorithm to Identify Confusable Drug Names, based on Bi-gram algorithms and the blend of the longest common subsequences. Furthermore, the research work achieved (S Al-Hagree et al. 2019) proposed an enhanced N-DIST method that concentrated on improving an algorithm for Name Matching. However, the previous studies differ from the contribution in this paper, because the proposed algorithm in this paper combines a Bi-gram technique with a distance technique (Salah Al-Hagree et al. 2019).

\section{The Proposed Method}

In this section, a Soft-Bidist is presented. The Soft-Bidist measure is an extension of BI-DIST, it softening the scale of comparison among the name Bigrams for improving the name detection. This section organizes as follows. The first subsection is to describe the involved cases of bigrams

${ }^{2}$ https://confluence.csiro.au/public/taxamatch/the-mdld-modified-damerau-levenshtein-distance-algorithm 
141 in the scale of the Soft-Bidist distance. Then, the Minimum, Maximum, and Average functions, 142 which are used as means to identify the weights in the distance scale by statistical means, are 143 mentioned. It is thus assumed that an evolutionary approach identifies the best levels in the distance 144 scale compared to the original distance scale that Kondrak proposed in BI-DIST (cf. Eqs. (3) and 145 (4)). In other words, we consider this problem as an evolutionary approach for optimizing the 146 internal parameters of the distance scale.

\section{Definition of Soft-Bidist Distance}

148 Let $\mathrm{X}$ and $\mathrm{Y}$ be given names represented as sequences of sizes $\mathrm{n}$ and $\mathrm{m}$, respectively, Soft-Bidist 149 is defined as follows:

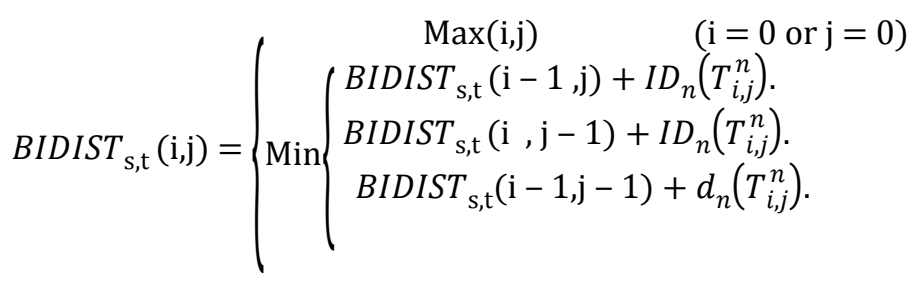

151

152

153

154

155

156

157

158

159

160

161

162

163

164

165

166

167

The distance scale for Soft-Bidist is shown as follows:

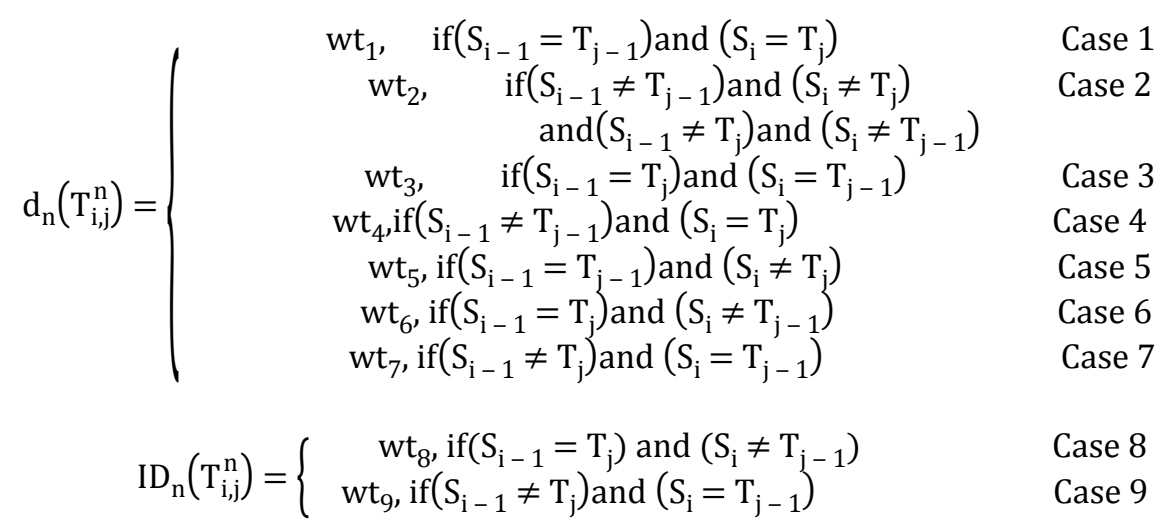

To increase the accuracy of identifying the names, there is a need to find the set of weights $\mathrm{WT}=\{\mathrm{wt} 1 ; \mathrm{wt} 2 ; \ldots ; \mathrm{wt} 9\}$ of the distance scale of Soft-Bidist. For this, a randomized value is used (Levenshtein 1966)(S Al-Hagree et al. 2019; Salah Al-Hagree et al. 2019; Earley 1969; Kondrak 2005; Millán-Hernández et al. 2019; Rees 2014).

\section{Definition of Soft-Bidist Distance}

The cases are weighted as symbols wt1, wt2, wt3, wt4, wt5, wt6, wt7, wt8, and wt9. These weights are depend on Table 1 and Table 2, which are used to adapt to the operational environment and get highly accurate results in various situations. Therefore, Table 1 contains several different weights. After changing the default values of $[0,1,1,0,1,1,1,1$ and 1] with wt1, wt2, wt3, wt4, wt5, wt6, wt7, wt8, and wt9 for all cases respectively, the new weights achieve results similar to that obtained by LD algorithm. Again, other default values have been examined $[0,1,0,0,1,1,1$, 1 and 1] with wt1, wt2, wt3, wt4, wt5, wt6, wt7, wt8 and wt9 for all cases respectively, the new 
168

169

170

171

172

173

174

175

176

177

178

179

180

181

182

183

184

185

186

187

188

189

190

191

192

193

194

195

196

197

198

199

200

201

202

203

204

205

206

207

weights achieve results similar to that obtained by the DLD algorithm. Finally, other default values of $[0,1,1,0.5,0.5,1,1,1$ and 1] for wt1, wt2, wt3, wt4, wt5, wt6, wt7, wt8, and wt9 for all cases respectively, the new weights achieves results similar to that obtained by the N-DIST algorithm. Based on the previous weight values, new weights were added to Table 2.

Table 1: The various weights for Soft-Bidist that yelled similar results to other algorithms from the literature

Table 2: The Randomize of weights for Soft-Bidist algorithm

\section{The Experimental Results}

This section presents the experimental results that are carried out in this research. The objective of these experiments is to assess the Soft-Bidist algorithm compared with other algorithms from the literature.. Due to the absence of standard datasets for name matching, different multilingual datasets (English, Arabic, Portuguese) is used in the experiments carried out in this research. These datasets are presented by (S Al-Hagree et al. 2019), (Ahmed and Nürnberger 2009), (Rees 2014) and (Al-Sanabani and Al-Hagree 2015). Different spelling errors and typographical are included in these datasets. In our previous work, a modified algorithm was applied to drug names in English documents, but for current work, the Soft-Bidist is applied to the different datasets deals with personal names in Arabic, English and Portuguese. To our knowledge, there have been no previous reports of good performance on this combination of datasets with different languages.

The same data preparation used in (S Al-Hagree et al. 2019) is used in this research to be compared with the Soft-Bidist algorithm.

After defining the default values of $[0,1,1,0.2,0.2,1,1,1$ and 1] for wt1, wt2, wt3, wt4, wt5, wt6, wt7, wt8, and wt9 for all cases respectively, the proposed algorithm appears to have achieved high accurate results. Generally, it is not easy to provide accurate weights between pair source and target. In order to gain proper values for weights wt1, wt2, wt3, wt4, wt5, wt6, wt7, wt8, and wt9, the experiments with different weights for Table 1 and Table 2 of dataset 1 (S Al-Hagree et al. 2019) should be repeated. The results are presented in Table 3.

Table 3: The results with different weights for Soft-Bidist

The experiments are repeated on dataset 2 (Ahmed and Nürnberger 2009)(Al-Sanabani and Al-Hagree 2015) for the Soft-Bidist algorithms. Table 4 shows the result of this experiment. It can be noticed that the Soft-Bidist Algorithm functions better than the DLD, LD and N-DIST algorithms particularly being compared with names transposition such as the names that are shown in rows 3 and 4. Unlike DLD, LD, and N-DIST, the Soft-Bidist algorithm is sensitive to replacement as shown in rows 6 and 7. The Soft-Bidist Algorithm computes recurring letters, detection of errors, and deletion in a more proficient manner than DLD, LD, and N-DIST as they 
208

209

210

211

212

213

214

215

216

217

218

219

220

221

222

223

224

225

226

227

228

229

230

231

232

233

234

235

236

237

238

239

240

241

242

243

244

245

246 247

appear in rows $5,8,9,10,11,12,13$, and 14 . The Soft-Bidist algorithm exhibits a number of advantages over the DLD, LD, and N-DIST algorithms as aforementioned. Therefore, the SoftBidist algorithm functions well and gives a better accuracy compared with the DLD, LD, and NDIST algorithms for all pairs in dataset 2 as appears in Table 4 .

Table 4: Comparison between proposed algorithms.

Furthermore, more experiments are implemented with various datasets to prove the evidence of the ability of the Soft-Bidist algorithm. Ten datasets are chosen and implemented on the DLD, LD, N-DIST, MDLD, and Soft-Bidist algorithms as appears in Table 5. That demonstrates the evidence and ability of the Soft-Bidist algorithm in name matching.

In Table 5, the Soft-Bidist algorithm gets $93 \%$ and $90 \%$ while DLD, LD, N-DIST, and MDLD algorithms get $88 \%, 88 \%, 86 \%$, and $89 \%$, respectively. Therefore, the Soft-Bidist algorithm gives more accurate results than the DLD, LD, N-DIST, and MDLD algorithms for all datasets, because LD, DLD, N-DIST, and MDLD algorithms have not considered the transposition operations of Latin-based language especially the English language.

\author{
Table 5: The mean similarity of LD, DLD, N-DIST, MDLD and Soft-Bidist algorithms with a \\ different dataset.
}

\title{
Comparative Study for Soft-Bidist Algorithm and compared
} algorithms

The dataset used for comparison in this section has been extracted manually from the book of (Christen 2012). To clarify the way that string comparison functions approximate various similarity estimations when used for similar strings. Table 6 gives sample results when given names and surnames are compared for the Soft-Bidist algorithm and compared algorithms as well. The highest similarity is shown in bold, while the lowest is shown in italics. The similarity values in Table 6 are calculated based on chosen name pairs. Table 6 reflects how different string comparison functions produce various similarity approximates for the same name pairs. According to the given results, there are significant differences in the similarities approximated on the same pair. These functions have various characteristics concerning the average and the spread of the value of similarity. Methods as Winkler, Jaro, the compression-based comparison operates, and Soft-Bidist Algorithm gives the highest mean of similarity values. Whereas, the edit distance (ED), the longest common substring (LCS) comparison, and the q-gram ('n-gram') based functions (Ukkonen 1992) result in a much lower mean in the similarity values as can be seen in Table 6. 
248

249

250

\section{The Estimated Measure}

\section{2}

253

254

255

256

257

258

259

260

261

262

263

264

265

266

267

268

269

270

271

272

273

274

275

276

277

278

279

280

281

282 defined as: measure is defined by: one Arabic dataset).
Table 6: The average similarities for proposed weights and compared methods presented at

(Christen 2012)

The estimated measure is using the f-measure which is also called f-score. The name matching quality has proven to be effective (P Christen 2006) (Christen, 2006; Olson et al.,2008; Kolomvatsos et al.,2013), which is based on precision and recall. These metrics are used for classification tasks. They compare the predicted class of an item with the actual class, as shown in Table 7. Based on Table 7 and following (Kolomvatsos et al., 2013), precision and recall are

$$
\begin{aligned}
& \text { Precision }=\frac{\mathrm{TP}}{\mathrm{TP}+\mathrm{FP}} \\
& \text { Recall }=\frac{\mathrm{TP}}{\mathrm{TP}+\mathrm{FN}}
\end{aligned}
$$

Moreover, the F-measure is defined as the weighted combination of precision and recall. The F-

$$
\mathrm{F}-\text { Measure }=\frac{2 . \text { Precision. Recall }}{\text { Precision }+ \text { Recall }}
$$

Table 7: Correspondence between the predicted and the actual classes.

Since f-measure is an accuracy measure between 0 and 1, the higher the values, the better and more accurate are the results. The experiments can be seen in Table 8 , the mean of f-measures achieved by the proposed Soft-Bidist algorithm on all instances for the used dataset and the threshold is 0.94, which outperforms the other algorithms. Best results shown boldface and worst results underlined. The thresholds are $0.90,0.85,0.80,0.75,0.70$ and 0.65 of all datasets tested (three English datasets, one Portuguese dataset, three species datasets, three genera datasets, and

Table 9 presents the F1-scores for different scenarios. For the dataset 5 (Portuguese 120 pairs), using different Edit Distance. The best results were retrieved with the threshold values for a correct 
283

284

285

286

287

288

289

290

291

292

293

294

295

296

297

298

299

300

301

302

303

304

305

306

307

308

309

310

311

312

313

314

315

316

317 318

319

320

match of $0.65,0.70,0.75,0.80,0.85$ and 0.90 for LD, DLD, N-DIST, MDLD and Soft-Bidist, respectively (Abdulhayoglu et al., 2016.). Table 9 shows F-measure vs. Threshold curves for dataset 5 (Portuguese 120 pairs).

Table 9: F1-scores of different algorithms, thresholds and similarity calculation.

Repeating the previous experiment has been carried based on all Datasets Table 10.the proposed algorithms Soft-Bidist $(0,1,0,0.2,0.2,1,1,1$ and 1$)$ and Soft-Bidist $(0,1,0,0.2,0.2,1,1,0.5$ and 0.5$)$ gives more accurate results than the algorithms LD,DLD,N-DIS and MDLD for all datasets as shown in Table 10. The mean of f-measures on all datasets as can be seen in Table 10, which equals 0.97 , says that accuracy is almost high and reasonable to trust the results. Best results have shown boldface and worst results underlined. The thresholds are 0.90, 0.85, 0.80, 0.75, 0.70 and 0.65 of all datasets tested (three English datasets, one Portuguese dataset, three species datasets, three genera datasets, and one Arabic dataset).

Table 10: The results of F-measure mean values

\section{Conclusion}

In this research, Soft-Bidist is proposed where it used a new methodology for improving namematching accuracy. Soft-Bidist algorithm handles the transposition, deletion, substitution, and insertion operations in a new way. These operations are dealt with differently, considering its different states of the name matching to enhance the matching performance. Furthermore, different weights were assigned for each operation, which in turn enhanced the whole matching process. In comparison with other algorithms from the literature, the results of the experiments prove that the Soft-Bidist outperformed compared algorithms significantly. For future studies, it is suggested to explore the evolutionary algorithms to get the most proper weights for the soft calculation case, Genetic Algorithm (GA) for instance.

\section{Funding}

Deanship of Scientific Research, Qassim University funding the publication of this project.

\section{Acknowledgments}

Researchers would like to thank the Deanship of Scientific Research, Qassim University for funding publication of this project. 


\section{Conflicts of Interest}

322 The authors declare no conflict of interest.

323

324

325

326

327

328

329

330

331

332

333

334

335

336

337

338

339

340

341

342

343

344

345

346

347

348

349

350

351

352

353

354

355

356

357

358

359

360

361

362

363

364

365

\section{References}

Abdulhayoglu, Mehmet Ali, Bart Thijs, and Wouter Jeuris. 2016. "Using Character N-Grams to Match a List of Publications to References in Bibliographic Databases." Scientometrics. doi: 10.1007/s11192-016-2066-3.

Ahmed, Farag, and Andreas Nürnberger. 2009. "Evaluation of N-Gram Conflation Approaches for Arabic Text Retrieval." Journal of the American Society for Information Science and Technology. doi: 10.1002/asi.21063.

Al-Hagree, S, M. Al-Sanabani, M. Hadwan, and M. A. Al-Hagery. 2019. "An Improved N-Gram Distance for Names Matching." Pp. 1-7 in 2019 First International Conference of Intelligent Computing and Engineering (ICOICE). IEEE.

Al-Hagree, Salah, Maher Al-Sanabani, Khaled M. A. Alalayah, and Mohammed Hadwan. 2019. "Designing an Accurate and Efficient Algorithm for Matching Arabic Names." in 2019 1st International Conference of Intelligent Computing and Engineering: Toward Intelligent Solutions for Developing and Empowering our Societies, ICOICE 2019.

Al-Sanabani, Maher, and Salah Al-Hagree. 2015. "Improved An Algorithm For Arabic Name Matching." OPEN TRANSACTIONS ON INFORMATION PROCESSING 1-17.

Al-Ssulami, Abdulrakeeb M. 2015. "Hybrid String Matching Algorithm with a Pivot." Journal of Information Science. doi: 10.1177/0165551514555668.

Berger, Bonnie, Michael S. Waterman, and Yun William Yu. 2020. "Levenshtein Distance, Sequence Comparison and Biological Database Search." IEEE Transactions on Information Theory. doi: 10.1109/tit.2020.2996543.

Charras, Christian, and Thierry Lecroq. 2004. Handbook of Exact String Matching Algorithms. King's College Publications.

Chowdhury, S. Dutta, U. Bhattacharya, and S. K. Parui. 2013. "Online Handwriting Recognition Using Levenshtein Distance Metric." in Proceedings of the International Conference on Document Analysis and Recognition, ICDAR.

Christen, P. 2006. A Comparison of Personal Name Matching Techniques and Practical Issues. edited by Joint Computer Science Technical Report Series. Department of Computer Science,

Christen, Peter. 2006. "A Comparison of Personal Name Matching: Techniques and Practical Issues." in Proceedings - IEEE International Conference on Data Mining, ICDM.

Christen, Peter. 2012. Data Matching: Concepts and Techniques for Record Linkage, Entity Resolution, and Duplicate Detection.

Damerau, Fred J. 1964. "A Technique for Computer Detection and Correction of Spelling Errors." Communications of the ACM. doi: 10.1145/363958.363994.

Delgado, Juan, Fernando Galárraga, Walter Fuertes, Theofilos Toulkeridis, Cesar Villacís, and Fidel Castro. 2016. "A Proposal of an Entity Name Recognition Algorithm to Integrate Governmental Databases." in 2016 3rd International Conference on eDemocracy and eGovernment, ICEDEG 2016.

Earley, Jay. 1969. 6. DAMERAU, F. A Technique for Computer Detection and Correc-Tion of Spelling Errors.

Hall, Patrick A. V., and Geoff R. Dowling. 1980. "Approximate String Matching.” $A C M$ 
366

367

368

369

370

371

372

373

374

375

376

377

378

379

380

381

382

383

384

385

386

387

388

389

390

391

392
Computing Surveys (CSUR). doi: 10.1145/356827.356830.

Hand, David, and Peter Christen. 2018. "A Note on Using the F-Measure for Evaluating Record Linkage Algorithms." Statistics and Computing. doi: 10.1007/s11222-017-9746-6.

Kondrak, Grzegorz. 2005. "N-Gram Similarity and Distance." in Lecture Notes in Computer Science (including subseries Lecture Notes in Artificial Intelligence and Lecture Notes in Bioinformatics).

Lertnattee, Verayuth, and Thitima Paluekpet. 2019. "Effects of Substring Position and Character Similarity on Human Perception of Thai Herb Name Similarity." in Lecture Notes in Electrical Engineering.

Levenshtein, Vladimir. 1966. "Binary Codes Capable of Correcting Deletions, Insertions, and Reversals." Soviet Physics Doklady.

Lisbach, Bertrand, Victoria Meyer, Bertrand Lisbach, and Victoria Meyer. 2013. "Name Matching and Identity Matching." in Linguistic Identity Matching.

Millán-Hernández, Christian Eduardo, René Arnulfo García-Hernández, Yulia Ledeneva, and Ángel Hernández-Castañeda. 2019. "Soft Bigram Similarity to Identify Confusable Drug Names." in Lecture Notes in Computer Science (including subseries Lecture Notes in Artificial Intelligence and Lecture Notes in Bioinformatics).

Navarro, Gonzalo. 2001. "A Guided Tour to Approximate String Matching." ACM Computing Surveys. doi: $10.1145 / 375360.375365$.

Park, Chanjun, Kuekyeng Kim, Yeong Wook Yang, Minho Kang, and Heuiseok Lim. 2020. "Neural Spelling Correction: Translating Incorrect Sentences to Correct Sentences for Multimedia." Multimedia Tools and Applications. doi: 10.1007/s11042-020-09148-2.

Rees, Tony. 2014. "Taxamatch, an Algorithm for near ('Fuzzy') Matching of Scientific Names in Taxonomic Databases.” PLoS ONE. doi: 10.1371/journal.pone.0107510.

Ukkonen, Esko. 1992. "Approximate String-Matching with q-Grams and Maximal Matches." Theoretical Computer Science. doi: 10.1016/0304-3975(92)90143-4. 


\section{Table $\mathbf{1}$ (on next page)}

The various weights for Soft-Bidist 
Table 1: The various weights for Soft-Bidist that yelled similar results to other algorithms from literature 1

\begin{tabular}{|c|c|c|c|c|c|c|c|c|c|}
\hline Proposed Weights & $\mathrm{wt}_{1}$ & $\mathrm{wt}_{2}$ & $\mathrm{wt}_{3}$ & $\mathrm{wt}_{4}$ & $\mathrm{wt}_{5}$ & $\mathrm{wt}_{6}$ & $\mathrm{wt}_{7}$ & $\mathrm{wt}_{8}$ & $\mathrm{wt}_{9}$ \\
\hline (LD). & 0 & 1, & 1, & 0 & 1 , & 1 & 1 & 1, & 1 \\
\hline (DLD). & 0 & 1 & 0 & 0 & 1, & 1, & 1 & 1 & 1 , \\
\hline $\mathrm{N}$-DIST is $\mathrm{n}=2$ "BI" & 0 & 1 & 1 & 0.5 & 0.5 & 1, & 1 & 1 & 1 \\
\hline
\end{tabular}




\section{Table 2 (on next page)}

The Randomize of weights for Soft-Bidist algorithm 
Table 2: The randomize of weights for Soft-Bidist algorithm

\begin{tabular}{|c|c|c|c|c|c|c|c|c|c|c|}
\hline No & Weights for Soft-Bidist & $\mathrm{wt}_{1}$ & $\mathrm{wt}_{2}$ & $\mathrm{wt}_{3}$ & $\mathrm{wt}_{4}$ & $\mathrm{wt}_{5}$ & $\mathrm{wt}_{6}$ & $\mathrm{wt}_{7}$ & $\mathrm{wt}_{8}$ & $\mathrm{wt}_{9}$ \\
\hline 1 & Minimum. & 0 & 1 & 0 & 0 , & 0.5 & 1 , & 1 , & 1 & 1 \\
\hline 2 & Average. & 0 & 1 & 0.7 & 0.2 & 0.8 & 1 , & 1 , & 1 & 1 \\
\hline 3 & Maximum (Cases 8,9 is 0.5 ). & 0 & 1 & 0 & 0 , & 0.5 & 1 , & 1 , & 0.5 & 0.5 \\
\hline 4 & Average (Cases 8,9 is 0.5$)$ & 0 & 1 & 0.7 & 0.2 & 0.8 & 1, & 1, & 0.5 & 0.5 \\
\hline 5 & & 0 & 1 & 1, & 0 , & 0.5 & 1 , & 1, & 1 & 1 \\
\hline 6 & & 0 , & 1, & 0 , & 0 , & 0.5 & 1, & 1, & 1 & 1 \\
\hline 7 & & 0 , & 1, & 0 & 0.2 & 0.2 & 1, & 1, & 1 & 1 \\
\hline 8 & 해요 & 0 , & 1, & 0 & 0.1 & 0.1 & 1, & 1, & 1 & 1 \\
\hline 9 & 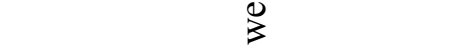 & 0 & 1, & 0 & 0 , & 0.2 & 1, & 1, & 1 & 1 \\
\hline 10 & $\stackrel{\mathbb{N}}{.}$ & 0 & 1, & 1 & 0 , & 0.5 & 1, & 1 & 0.5 & 0.5 \\
\hline 11 & ్ㅡㅁ & 0 & 1, & 0.5 & 0 , & 0.5 & 1, & 1, & 0.5 & 0.5 \\
\hline 12 & ]్ & 0 & 1, & 0 & 0.1 & 0.1 , & 1, & 1, & 0.5 & 0.5 \\
\hline 13 & & 0 , & 1 , & 0.5 & 0 , & 0.1 & 1, & 1, & 0.5 & 0.5 \\
\hline 14 & & 0 , & 1, & 0 & 0 , & 0.2 & 1, & 1, & 0.5 & 0.5 \\
\hline 15 & & 0 , & 1, & 0 & 0 , & 0.1, & 1, & 1, & 0.5 & 0.5 \\
\hline 16 & The applied in this paper. & 0 & 1 , & 0 & 0.2 & 0.2 & 1 , & 1 , & 0.5 & 0.5 \\
\hline
\end{tabular}




\section{Table 3 (on next page)}

The results with different weights for Soft-Bidist 
Table 3: The results with different weights for Soft-Bidist

\begin{tabular}{clc}
\hline No & $\mathrm{wt}_{1}, \mathrm{wt}_{2}, \mathrm{wt}_{3}, \mathrm{wt}_{4}, \mathrm{wt}_{5}, \mathrm{wt}_{6}, \mathrm{wt}_{7}, \mathrm{wt}_{8}$ and $\mathrm{wt}_{9}$ & $\begin{array}{c}\text { Average } \\
\text { (percentage } \\
\text { similarity) }\end{array}$ \\
\hline 1 & $0,1,1,0,1,1,1,1$ and 1 & 0.83 \\
2 & $0,1,0,0,1,1,1,1$ and 1 & 0.87 \\
3 & $0,1,1,0.5,0.5,1,1,1$ and 1 & 0.82 \\
4 & $0,1,0,0.5,0.5,1,1,1$ and 1 & 0.89 \\
5 & $0,1,1,0,0.5,1,1,1$ and 1 & 0.87 \\
6 & $0,1,0,0,0.5,1,1,1$ and 1 & 0.91 \\
7 & $0,1,0,0.2,0.2,1,1,1$ and 1 & 0.91 \\
8 & $0,1,0,0.1,0.1,1,1,1$ and 1 & 0.93 \\
9 & $0,1,0,0,0.2,1,1,1$ and 1 & 0.93 \\
10 & $0,1,1,0,0.5,1,1,0.5$ and 0.5 & 0.89 \\
11 & $0,1,0.5,0,0.5,1,1,0.5$ and 0.5 & 0.91 \\
12 & $0,1,0,0,0.5,1,1,0.5$ and 0.5 & 0.93 \\
13 & $0,1,0.5,0,0.5,1,1,0.5$ and 0.5 & 0.88 \\
14 & $0,1,0,0.2,0.2,1,10.5$ and 0.5 & $\mathbf{0 . 9 4}$ \\
15 & $0,1,0,0.1,0.1,1,1,0.5$ and 0.5 & 0.96 \\
16 & $0,1,0.5,0,0 ., 1,1,0.5$ and 0.5 & 0.95 \\
17 & $0,1,0,0,0.2,1,1,0.5$ and 0.5 & 0.96 \\
18 & $0,1,0,0,0.1,1,1,0.5$ and 0.5 & 0.97 \\
\hline
\end{tabular}




\section{Table 4 (on next page)}

Comparison between algorithm from literature and Soft-Bidist 
Table 4: Comparison between proposed algorithms and compared algorithm.

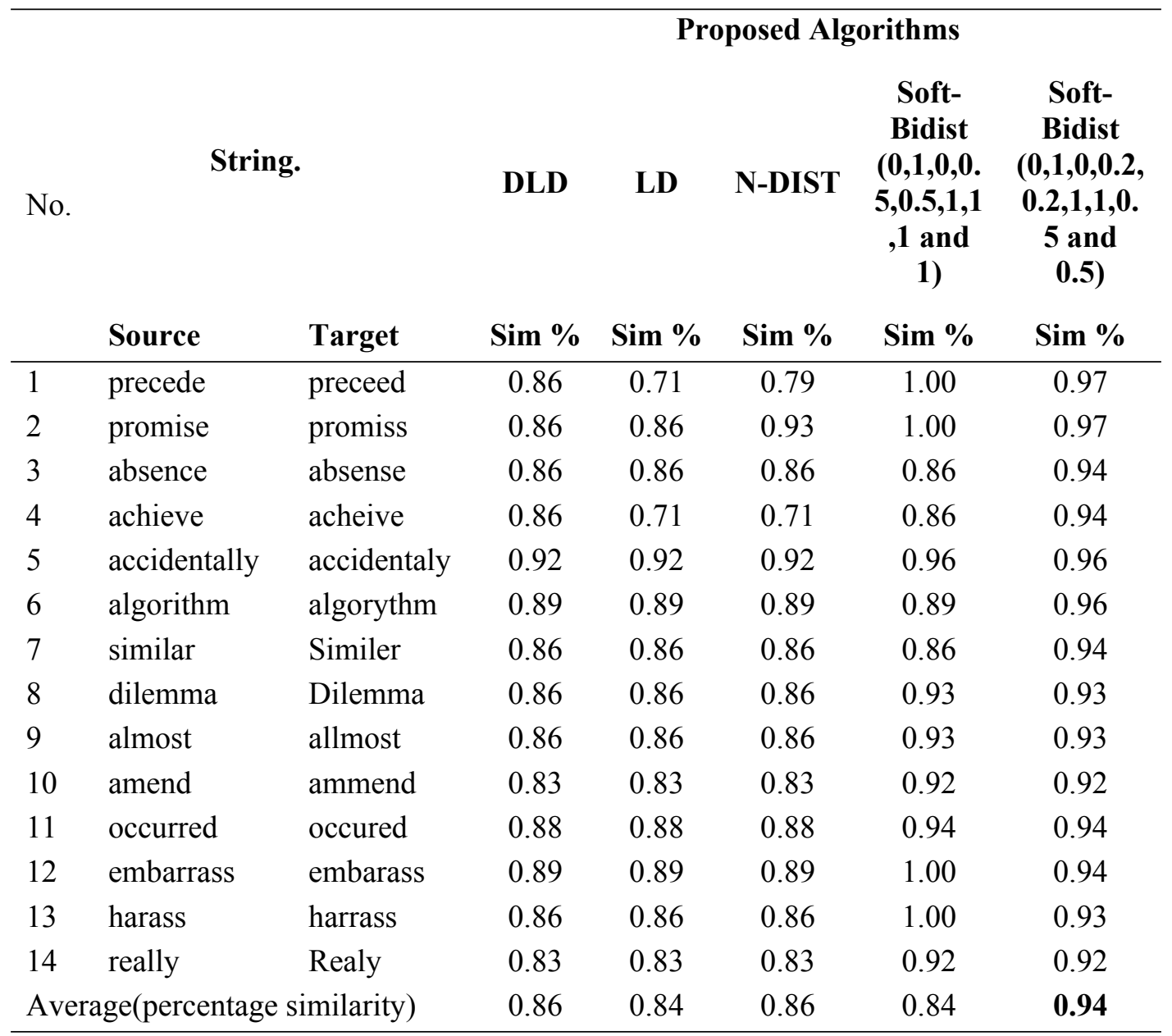




\section{Table 5 (on next page)}

The mean similarity of LD, DLD, N-DIST, MDLD and Soft-Bidist algorithms with different dataset. 
Table 5: The mean similarity of LD, DLD, N-DIST, MDLD and Soft-Bidist algorithms with different 1 dataset.

\begin{tabular}{|c|c|c|c|c|c|c|c|}
\hline \multicolumn{2}{|c|}{ Datasets } & \multirow{2}{*}{$\begin{array}{r}\text { DLD } \\
\text { Sim \% }\end{array}$} & \multirow{2}{*}{$\begin{array}{r}\text { LD } \\
\text { Sim \% }\end{array}$} & \multirow{2}{*}{$\begin{array}{l}\text { N-DIST } \\
\text { Sim \% }\end{array}$} & \multirow{2}{*}{$\begin{array}{l}\text { MDLD } \\
\text { Sim \% }\end{array}$} & \multirow{2}{*}{$\begin{array}{c}\text { Soft-Bidist } \\
(0,1,0,0.5,0.5 \text {, } \\
1,1,1 \text { and } 1) \\
\text { Sim \% }\end{array}$} & \multirow{2}{*}{$\begin{array}{c}\text { Soft-Bidist } \\
(0,1,0,0.2,0.2,1 \\
, 1,0.5 \text { and } 0.5) \\
\text { Sim \% }\end{array}$} \\
\hline & & & & & & & \\
\hline 1 & $\begin{array}{l}\text { Dataset } 3 \text { (English } 60 \\
\text { pairs) [19]. }\end{array}$ & 0.86 & 0.83 & 0.81 & 0.87 & 0.89 & 0.94 \\
\hline 2 & $\begin{array}{l}\text { Dataset } 4 \text { (English } 4013 \\
\text { pairs) [19]. }\end{array}$ & 0.85 & 0.84 & 0.82 & 0.86 & 0.89 & 0.92 \\
\hline 3 & $\begin{array}{l}\text { Dataset5 (Portuguese } \\
120 \text { pairs) [21]. }\end{array}$ & 0.84 & 0.84 & 0.82 & 0.84 & 0.85 & 0.91 \\
\hline 4 & $\begin{array}{l}\text { Dataset } 6 \text { 'CAAB' (641 } \\
\text { pairs) [16]. }\end{array}$ & 0.94 & 0.95 & 0.93 & 0.94 & 0.95 & 0.96 \\
\hline 5 & $\begin{array}{l}\text { Dataset } 7 \text { 'Dalcin name } \\
\text { pairs' (171 pairs) [16]. }\end{array}$ & 0.95 & 0.94 & 0.93 & 0.95 & 0.97 & 0.97 \\
\hline 6 & $\begin{array}{l}\text { Dataset } 8 \text { 'CAABWEB' } \\
\text { (2047 pairs) [16]. }\end{array}$ & 0.93 & 0.93 & 0.92 & 0.93 & 0.95 & 0.95 \\
\hline 7 & $\begin{array}{l}\text { Dataset } 9 \text { 'GRIN genera' } \\
\text { (189 pairs) [16]. }\end{array}$ & 0.89 & 0.88 & 0.87 & 0.89 & 0.90 & 0.94 \\
\hline 8 & $\begin{array}{l}\text { Dataset } 10 \text { 'CAAB } \\
\text { Genera' (115 pairs) [16]. }\end{array}$ & 0.90 & 0.88 & 0.85 & 0.90 & 0.91 & 0.94 \\
\hline 9 & $\begin{array}{l}\text { Dataset } 11 \text { 'CAABWEB } \\
\text { Genera' ( } 853 \text { pairs) [16]. }\end{array}$ & 0.88 & 0.88 & 0.87 & 0.88 & 0.90 & 0.93 \\
\hline 10 & $\begin{array}{l}\text { Dataset } 12 \text { 'Arabic name } \\
\text { (600 pairs) [22]. }\end{array}$ & 0.80 & 0.79 & 0.73 & 0.80 & 0.77 & 0.80 \\
\hline & Similarity mean & 0.88 & 0.88 & $\underline{0.86}$ & 0.89 & 0.90 & 0.93 \\
\hline
\end{tabular}




\section{Table 6(on next page)}

Similarities of calculated and string pairs 
Table 6: The average similarities for proposed weights and compared methods presented at (Christen 2012)

\begin{tabular}{llr}
\hline No. & Algorithms & $\begin{array}{r}\text { Average } \\
\text { similarity }\end{array}$ \\
\hline 1 & Jaro & 0.86 \\
2 & Winkler & $\mathbf{0 . 8 8}$ \\
3 & Bigram & 0.62 \\
4 & Trigram & 0.52 \\
5 & Positional bigrams & 0.62 \\
6 & Skip-grams & 0.62 \\
7 & Levenshtein edit distance (LD) & 0.70 \\
8 & Damerau-Levenshtein edit distance (DLD) & 0.72 \\
9 & BagDist & 0.78 \\
10 & Editex & 0.75 \\
11 & compression-based similarity using the ZLib compressor & 0.66 \\
12 & longest common substring (length =2) & 0.67 \\
13 & longest common substring (length =3) & 0.60 \\
14 & Smith-Waterman edit distance & 0.65 \\
15 & syllable alignment distance & 0.66 \\
16 & MDLD & 0.72 \\
17 & $0,1,1,0,1,1,1,1$ and 1(Sof-Bidist) & 0.70 \\
18 & $0,1,0,0,1,1,1,1$ and 1 (Sof-Bidist) & 0.72 \\
19 & $0,1,1,0.5,0.5,1,1,1$ and 1 (Sof-Bidist) & 0.68 \\
20 & $0,1,0,0.5,0.5,1,1,1$ and 1 (Sof-Bidist) & 0.77 \\
21 & $0,1,0,0.2,0.2,1,1,1$ and 1 (Sof-Bidist) & 0.78 \\
22 & $0,1,0,0.1,0.1,0.5,0.5,0.5$ and 0.5 (Sof-Bidist) & 0.83 \\
23 & $0,1,0,0,0.1,0.5,0.5,0.5$ and 0.5 (Sof-Bidist) & 0.85 \\
24 & $0,1,0,0,0.1,0.2,0.2,0.2$ and 0.2 (Sof-Bidist) & $\mathbf{0 . 8 8}$ \\
\hline & &
\end{tabular}




\section{Table 7 (on next page)}

Correspondence between the predicted and the actual classes 
Table 7: Correspondence between the predicted and the actual classes.

\begin{tabular}{c|ccc}
\hline \hline \multicolumn{2}{c}{ Algorithm } & \multicolumn{2}{c}{ Predicted } \\
\cline { 3 - 4 } Actual & Match & True Positive (TP) & False Negative (FN) \\
(Truth) & Not Match & False Positive (FP) & True Negative (TN) \\
\hline \hline
\end{tabular}




\section{Table 8(on next page)}

Table 8: Average f-measure values (best results shown boldface and worst results underlined) with threshold $0.90,0.85,0.80,0.75,0.70$ and 0.65 , of all datasets tested ( 3 for English , 1 for portuguese, 3 for species, 3 for genera , 1 for Arabic ). 
Table 8: The results of average f-measure values

\begin{tabular}{|c|c|c|c|c|c|c|c|}
\hline & \multirow{3}{*}{ Datasets } & \multicolumn{4}{|c|}{ Compared Algorithm } & \multicolumn{2}{|c|}{ Proposed Algorithm } \\
\hline & & LD & DLD & $\begin{array}{l}\text { N- } \\
\text { DIST }\end{array}$ & MDLD & $\begin{array}{c}\text { Soft-Bidist } \\
(0,1,0,0.5,0.5 \\
1,1,1 \text { and } 1)\end{array}$ & $\begin{array}{c}\text { Soft-Bidist } \\
(0,1,0,0.2,0.2, \\
1,1,0.5 \text { and } \\
0.5)\end{array}$ \\
\hline & & $\operatorname{Sim} \%$ & $\operatorname{Sim} \%$ & $\operatorname{Sim} \%$ & $\operatorname{Sim} \%$ & Sim \% & $\operatorname{Sim} \%$ \\
\hline 1 & $\begin{array}{l}\text { Dataset } 3 \text { ( English } \\
60 \text { pairs) }\end{array}$ & 0.77 & 0.85 & $\underline{0.74}$ & 0.85 & 0.91 & 0.95 \\
\hline 2 & $\begin{array}{l}\text { Dataset } 4 \text { ( English } \\
4013 \text { pairs) } \\
\text { Dataset } 5 \text { ( }\end{array}$ & 0.75 & 0.76 & $\underline{0.73}$ & 0.89 & 0.90 & 0.94 \\
\hline 3 & $\begin{array}{l}\text { Portuguese } 120 \\
\text { pairs) }\end{array}$ & 0.80 & 0.80 & $\underline{0.77}$ & 0.80 & 0.82 & 0.93 \\
\hline 4 & $\begin{array}{l}\text { Dataset } 6 \text { 'CAAB' } \\
\text { ( } 641 \text { pairs) }\end{array}$ & 0.99 & 0.99 & 0.99 & 0.99 & 1.00 & 1.00 \\
\hline 5 & $\begin{array}{l}\text { Dataset } 7 \text { 'Dalcin } \\
\text { name pairs' }(171 \\
\text { pairs) } \\
\text { Dataset } 8\end{array}$ & 1.00 & 1.00 & 1.00 & 1.00 & 1.00 & 1.00 \\
\hline 6 & $\begin{array}{l}\text { 'CAABWEB' (2047 } \\
\text { pairs) }\end{array}$ & 0.96 & 0.97 & 0.94 & 0.98 & 0.98 & 0.99 \\
\hline 7 & $\begin{array}{l}\text { Dataset } 9 \text { 'GRIN } \\
\text { genera' (189 pairs) }\end{array}$ & 0.93 & 0.94 & $\underline{0.88}$ & 0.94 & 0.95 & 0.95 \\
\hline 8 & $\begin{array}{l}\text { Dataset } 10 \text { 'CAAB } \\
\text { Genera' (115 pairs) } \\
\text { Dataset } 11\end{array}$ & 0.95 & 0.96 & $\underline{0.88}$ & 0.96 & 0.97 & 0.96 \\
\hline 9 & $\begin{array}{l}\text { 'CAABWEB } \\
\text { Genera' ( } 853 \text { pairs) }\end{array}$ & 0.91 & 0.93 & $\underline{0.84}$ & 0.79 & 0.91 & 0.94 \\
\hline 10 & $\begin{array}{l}\text { Dataset } 1 \text { 'Arabic } \\
\text { name ( } 600 \text { pairs) }\end{array}$ & 0.66 & 0.68 & $\underline{0.53}$ & 0.68 & 0.70 & 0.77 \\
\hline & $\begin{array}{l}\text { F-MEASURE } \\
\text { MEAN }\end{array}$ & 0.87 & 0.89 & $\underline{0.83}$ & 0.89 & 0.91 & 0.94 \\
\hline
\end{tabular}




\section{Table 9 (on next page)}

Table 9: F1-scores of different algorithms, thresholds and similarity calculation. 
Table 9: F1-scores of different algorithms, thresholds and similarity calculation.

\begin{tabular}{llcccccc}
\hline \hline & & & & & & & \\
\end{tabular}




\section{Table $\mathbf{1 0}$ (on next page)}

Table 10: F-measure mean values (best results shown boldface and worst results underlined) with threshold $0.90,0.85,0.80,0.75,0.70$ and 0.65 , of all datasets tested (3 for English , 1 for Portuguese, 3 for species, 3 for genera , 1 for Arabic ). 
Table 10: The results of F-measure mean values

\begin{tabular}{|c|c|c|c|c|c|c|c|}
\hline & \multirow{3}{*}{ Datasets } & \multicolumn{4}{|c|}{ Compared Algorithm } & \multicolumn{2}{|c|}{ Proposed Algorithm } \\
\hline & & LD & DLD & N-DIST & MDLD & $\begin{array}{c}\text { Soft-Bidist } \\
(0,1,0,0.2,0( \\
.2,1,1,0.5 \\
\text { and } 0.5)\end{array}$ & $\begin{array}{c}\text { Soft-Bidist } \\
(0,1,0,0.2,0.2, \\
1,1,0.5 \text { and } \\
0.5)\end{array}$ \\
\hline & & $\operatorname{Sim} \%$ & $\operatorname{Sim} \%$ & $\operatorname{Sim} \%$ & $\operatorname{Sim} \%$ & Sim $\%$ & $\operatorname{Sim} \%$ \\
\hline 1 & $\begin{array}{l}\text { Dataset } 3 \text { ( English } \\
60 \text { pairs) }\end{array}$ & 0.77 & 0.85 & $\underline{0.74}$ & 0.85 & 0.95 & 1.00 \\
\hline 2 & $\begin{array}{l}\text { Dataset } 4 \text { ( English } \\
4013 \text { pairs) }\end{array}$ & 0.75 & 0.76 & $\underline{0.73}$ & 0.89 & 0.94 & 0.99 \\
\hline 3 & $\begin{array}{ll}\text { Dataset } & 5 \\
\text { Portuguese } & 120 \\
\text { pairs) } & \end{array}$ & 0.80 & 0.80 & $\underline{0.77}$ & 0.80 & 0.93 & 0.95 \\
\hline 4 & $\begin{array}{l}\text { Dataset } 6 \text { 'CAAB' ( } \\
641 \text { pairs) }\end{array}$ & 0.99 & 0.99 & 0.99 & 0.99 & 1.00 & 1.00 \\
\hline 5 & $\begin{array}{l}\text { Dataset } 7 \\
\text { name pairs' } \\
\text { pairs })\end{array}$ & 1.00 & 1.00 & 1.00 & 1.00 & 1.00 & 1.00 \\
\hline 6 & $\begin{array}{lr}\text { Dataset } & 8 \\
\text { 'CAABWEB' } & (2047 \\
\text { pairs }) & \end{array}$ & 0.96 & 0.97 & 0.94 & 0.98 & 0.99 & 0.99 \\
\hline 7 & $\begin{array}{l}\text { Dataset } 99 \text { 'GRIN } \\
\text { genera' (189 pairs) }\end{array}$ & 0.93 & 0.94 & $\underline{0.88}$ & 0.94 & 0.95 & 0.99 \\
\hline 8 & $\begin{array}{l}\text { Dataset } 10 \text { 'CAAB } \\
\text { Genera' (115 pairs) }\end{array}$ & 0.95 & 0.96 & $\underline{0.88}$ & 0.96 & 0.96 & 0.99 \\
\hline & Dataset $\quad 11$ & & & & & & \\
\hline 9 & $\begin{array}{l}\text { 'CAABWEB Genera' } \\
\text { (853 pairs) }\end{array}$ & 0.91 & 0.93 & $\underline{0.84}$ & 0.79 & 0.94 & 0.98 \\
\hline 10 & $\begin{array}{l}\text { Dataset } 1 \text { 'Arabic } \\
\text { name ( } 600 \text { pairs) }\end{array}$ & 0.66 & 0.68 & $\underline{0.53}$ & 0.68 & 0.77 & 0.81 \\
\hline & F-MEASURE MEAN & 0.87 & 0.89 & $\underline{0.83}$ & 0.89 & 0.94 & 0.97 \\
\hline
\end{tabular}

